\title{
Ciliary function of the nose in patients with Osler's disease and the effect of topically applied estrogens as a nose ointment*
}

\author{
J. Ulrich Sommer, Boris A. Stuck, Clemens Heiser, Stefan S. Kassner, \\ K. Hörmann, Haneen Sadick
}

Department of Otorhinolaryngology Head and Neck Surgery, University Hospital Mannheim, Germany

\begin{abstract}
SUMMARY Background: In recent years, the positive effect of topically applied estriol nose ointment in the adjuvant therapy of Morbus-Rendu-Osler (HHT) has been proven. Due to the induced metaplasia, a complete destruction of the ciliated cells may be expected. However, data regarding the ciliary function of HHT patients with and without the use of topical estriol application are currently lacking.

Methodology/Principal: Ciliated samples were obtained by gently brushing the inferior nasal turbinate of 19 healthy volunteers and 15 patients with known HHT (8 of them regularly using $0.1 \%$ estriol nose ointment for 2 years (HHTwE) and 7 of them not using the ointment in the last 12 months (HHTwoE)). Analysis was done with an inverted phase contrast microscope connected to a high-speed digital camera. Recorded parameters were the visual integrity (VI) of the ciliary beat and its frequency $(C B F)$ in $\mathrm{Hz}$.

Results: The VI index of all samples showed an undisrupted, even beating pattern with difference between the three groups. The mean CBF in all HHT patients was reduced compared to the control group's mean CBF. Within the HHT group itself, the mean CBF was reduced in the HHTwE group compared to the HHTwoE group.

Conclusions: The ciliary beat frequency of HHT patients is impaired compared to the control group and even more so if the HHT patients topically apply estriol more than 6 months. An undisrupted beating pattern is found in the HHTwE group despite the fact that estrogens induce a transformation of the ciliated columnar into a keratinizing squamous epithelium. This data may justify the adjuvant application of estriol as a nose ointment in the treatment of epistaxis in HHT patients without the fear of damage to the nose's mucus clearance.
\end{abstract}

Key words: cilia, CBF, Morbus Osler, HHT, estriol

\section{INTRODUCTION}

The primary function of cilia in the upper respiratory tract is to eliminate dust and other inhaled particles. A fast and synchronous beating pattern is therefore necessary for optimal function. The debris is transported in a layer of mucus, much like a conveyor belt ${ }^{(1-3)}$. Not all factors that influence ciliary beat frequency (CBF) are understood ${ }^{(4)}$. Currently, temperature ${ }^{(5)}$, various chemical substances ${ }^{(6)}$ and infections ${ }^{(7)}$ are known to directly influence beat frequency. Toxic agents, like alcohol or nicotine, can permanently reduce $\mathrm{CBF}$ if taken over a long period of time ${ }^{(8)}$. An inherent dysfunction of motile cilia is the primary ciliary dyskinesia (PCD), an autosomal recessive disorder ${ }^{(9)}$.
The Osler-Weber-Rendu syndrome is a systemic fibrovascular dysplasia. It was first described at the end of the $19^{\text {th }}$ century (10-12) and is characterized by mucocutaneous telangiectasias and arteriovenous malformations. Therefore, the disease is also called hereditary hemorragic telangiectasia (HHT). The irregular blood vessels present an incomplete perivascular ring of smooth musculature with a brittle and fragile structure ${ }^{(13)}$ that leads to recurring episodes of epistaxis as one of the main symptoms.

The inheritance of HHT is autosomal dominant with a prevalence of approximately one in $500{ }^{(14)}$. Diagnosis of HHT is 
done clinically based on the Curaçao criteria and, according to the international guideline for the diagnosis and management of hereditary hemorrhagic telangiectasia, genetic testing ${ }^{(14,15)}$.

In the past, a variety of surgical and non-surgical approaches have been applied in the treatment of epistaxis in HHT. The use of a nasal packing is often found in the acute phase of epistaxis or in emergency care situations. Surgical treatment modalities include electrocauterization ${ }^{(16)}$ or chemical coagulation ${ }^{(17)}$, laser surgery ${ }^{(18)}$, argon plasma coagulation ${ }^{(16)}$, Saunders septodermoplasty ${ }^{(19,20)}$ and embolization ${ }^{(21)}$. If all treatment options fail, a closure of the nasal cavity known as Young's procedure has been described as ultima ratio to control nosebleeds ${ }^{(22)}$. These procedures are often combined with an adjuvant therapy that comprises the humidification of the nasal mucosa in form of nose ointments or inhalations. Some nose ointments described in literature not only provide humidification of the mucosa but also influence the nasal mucosa in such a way that it becomes less vulnerable to local trauma due to their special mixture and composition. Possible ingredients are antifibrinolytics for inhibition of blood clot disintegration ${ }^{(23)}$ or topically applied estriol, which is a low-potent metabolite of estrogen ${ }^{(24,25)}$. Estrogens are known to induce a metaplastic change of the nasal mucosa ${ }^{(26)}$. In our previous study published in 2005, we demonstrated that topically applied estriol as a nose ointment has the potency to induce a thick epithelial lining by changing the former columnar epithelium into a keratinizing squamous epithelium that covers the telangiectasia in the nasal cavity ${ }^{(26)}$. However, these treatment measures can negatively impact the ciliated epithelia of the nose due to the reduction of ciliated columnar epithelium with the risk of having a dry and crusted nose ${ }^{(25)}$.

To measure CBF, various methods have been developed. The saccharine transport time approach measures the time it takes for the beating cilia to transport particles like artificial sweeteners along the nasal mucosa ${ }^{27,28)}$. To analyze the beating cilia themselves, cilia have to be obtained directly from the nasal mucosa. A minimally invasive method of collecting ciliated cells from HHT patients is necessary to protect the vulnerable endonasal mucosal telangiectasias from trauma ${ }^{(13)}$ and to minimize the risk of bleeding. In these cases, gentle brush cytologies of the inferior nasal turbinate may be more appropriate despite the known drawbacks. For example, brushed cells are often disrupted or mechanically impaired, so only a limited number of cell clusters can be analyzed. Furthermore, the cells have to be kept in a nutrition solution for a certain time after harvesting, which potentially modifies their function ${ }^{(29)}$.

No data exist on the ciliary function and CBF of the nose in HHT patients and the influence of estrogen treatment despite the fact that estrogens have been in use for a long time as either a systemic or topical agent in the treatment of epistaxis in HHT patients and despite the fact that the ciliary function is crucial for the functional integrity of the nasal mucosa ${ }^{(30)}$.
With consecutive changes of the mucosal endonasal epithelium after long-term estriol application, one would assume that there is no ciliary function or at least reduced function in HHT patients.

In this new study, we were interested to find out (a) whether ciliary function is impaired in patients with HHT compared to healthy controls, (b) in what way the long-term administration of estriol as a nose ointment impacts the CBF, (c) whether the ultrastructure of the cilia-bearing cells remains intact and (d) whether application of estriol as a nose ointment is a safe treatment option for HHT patients.

\section{MATERIALS AND METHODS}

Study protocol

This study was performed at the Department of Otorhinolaryngology, Head and Neck Surgery at the University Hospital Mannheim. The study protocol was reviewed and approved by the local Ethics Board of the Medical Faculty Mannheim, University of Heidelberg. Written informed consent was obtained from all participants.

\section{Samples}

Ciliated samples were obtained by gently brushing the inferior nasal turbinate of 19 healthy volunteers. Eight patients were female and 11 patients were male with a mean age of 36 years (ranging from 25 to 56 years). Furthermore, 15 patients with known HHT were enrolled in this study. Five of these patients were female and 10 patients were male. The age ranged from 27 to 74 years with a mean age of 59 years. Within the HHT group, 8 patients regularly used $0.1 \%$ estriol nose ointment at least twice a day for the last two years. The other 7 HHT patients reported not to be using $0.1 \%$ estriol nose ointment at all for the last 12 months.

Brushing was done with a standard cytology brush (Gynobrush Plus, Heinz Herenz, Hamburg, Germany). Using anterior rhinoscopy, the wider nasal passage was determined and used for harvesting.

The cytology brush was dipped in $0.9 \%$ saline solution prior to taking the sample from the inferior turbinate. Immediately after brushing, the brush was submerged in $5 \mathrm{ml} \mathrm{RPMI}$ medium (RPMI 1640, cell culture tested, standard, L-Glutamine: 300 mg/l, PromoCell, Heidelberg, Germany).

\section{Microscopy}

The cytology brush was then shaken and the medium was stored at room temperature for 4 hours. During this time, the CBF slightly increased and then stabilized ${ }^{(31)}$. Subsequently, the medium was transferred to a Petri dish and placed under an inverted phase contrast microscope (Leica Microsystems $\mathrm{GmbH}$, Wetzlar, Germany). Magnification was set to 400x and a cluster of beating cells was selected. Only intact clusters were analyzed since disrupted cells generally show a significantly lower $\mathrm{CBF}{ }^{(32)}$. After acquiring a sharp, bright image, the light beam was directed into the SAVA system's high-speed digital 
camera (Ammons Engineering, Mt. Morris, MI, USA) ${ }^{(33)}$. A sequence of two seconds was recorded at a frame rate of 100 frames per second (fps). This process of identifying cell clusters and taking short video sequences was repeated up to 25 times, depending on how many beating cell clusters could be located in the sample. During microscopy, the temperature was monitored using an infrared pyrometer (Fluke 68 / Fluke GmbH, Kassel, Germany) and kept stable at about $22^{\circ} \mathrm{C} \pm 0.5^{\circ} \mathrm{C}$.

\section{Ciliary beat frequency analysis}

The CBF analysis was performed after finishing the clinical part of the study using the SAVA ${ }^{(33)}$ system's point of interest (POI) method. A rectangle was defined surrounding the cluster's beating edge. Within this rectangle the software assessed a recurring pattern of bright and dark changes. Figure 1 illustrates this process. Afterwards, the visual integrity of the sample's ciliary beat was recorded on a three-grade scale: grade 0 standing for no beating cilia or a heavily disrupted ciliary beating pattern in the samples, grade 1 representing samples with motile cilia and a moderately disrupted ciliary beating pattern and grade 3 indicating samples with a normal or hardly disrupted ciliary beating pattern. This three-grade scale was classified as the visual integrity index (VI index). The analysis and graduation of the tissue samples was performed by an external, blinded investigator.

\section{Statistics}

Statistical analysis and plotting was done using $\mathrm{R}$, an open source environment for statistical computing and graphics ${ }^{(34)}$. All data records were tested with the Shapiro-Wilk test and appeared to be normally distributed. The Mann-WhitneyWilcoxon rank sum test was used as a nonparametric test for statistical analysis of the CBF in HHT patients using estriol nose ointment versus the CBF in HHT patients not using estriol nose ointment. For the purpose of comparing the three-grade VI index as an ordinal scale, the Mann-Whitney-Wilcoxon rank sum test was used. A p-value less than 0.05 was considered statistically significant.

\section{RESULTS}

\section{Ciliary beating pattern}

The VI index in all 15 HHT patients showed an undisrupted, even ciliary beating pattern $(1.40 \pm 0.63)$ similar to the beating pattern of the control group $(\mathrm{n}=19)$ with a mean VI index of $1.58( \pm 0.51)$. No statistically significant difference could be found between both groups $(\mathrm{p}=0.54)$. Even within the HHT group itself, the VI index did not show a statistically significant difference $(\mathrm{p}=0.51)$ between the 8 HHT patients using the estriol nose ointment $(1.25 \pm 0.71)$ and the 7 HHT patients not using the estriol nose ointment $(1.57 \pm 0.53)$.

\section{Ciliary beat frequency $(C B F)$}

The mean CBF in all HHT patients was $4.75 \mathrm{~Hz}( \pm 2.23 \mathrm{~Hz})$, which is significantly reduced when compared to the mean $\mathrm{CBF}$ of the healthy control group at $9.12 \mathrm{~Hz}( \pm 2.43 \mathrm{~Hz})$, with a $\mathrm{p}<$

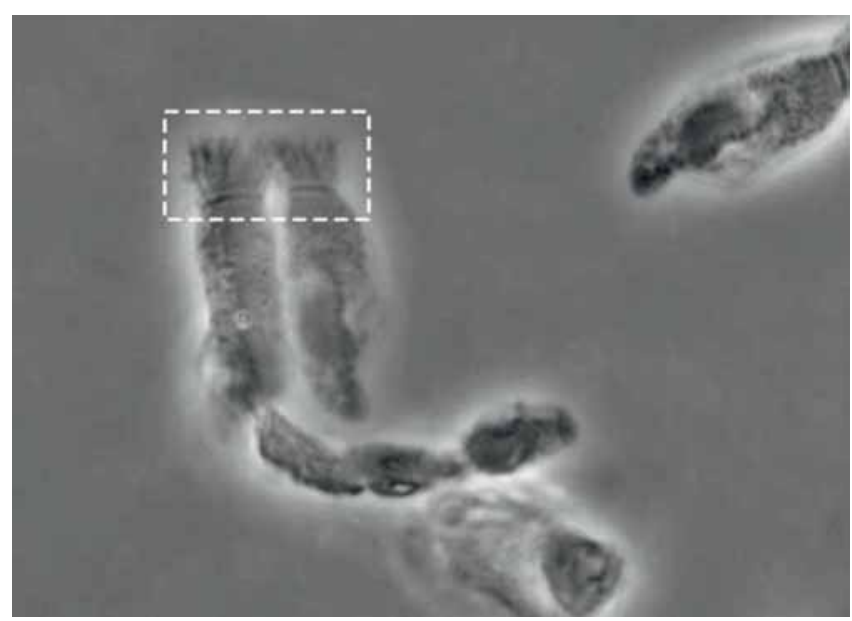

Figure 1. Beating cluster of ciliated cells. The rectangle with its beating cilia is selected using SAVA-software's 'POI-method.'

0.001 (Figure 2). Within the HHT group itself, the mean CBF was significantly reduced in HHT patients using the estriol ointment $(2.93 \mathrm{~Hz} \pm 0.6 \mathrm{~Hz})$ compared to the HHT patients not using the estriol ointment $(6.82 \mathrm{~Hz} \pm 1.33 \mathrm{~Hz})$, with $\mathrm{p}<0.001$. Figure 3 depicts the significant $\mathrm{CBF}$ difference in both the groups. Outliers in this figure were furthermore marked as circles. Note that the y-axis is not to the same scale as in Figure 2.

\section{DISCUSSION}

Ciliary beating pattern

In our previous study, we demonstrated through electron microscopic analysis of the nasal mucosa of HHT patients that the nasal epithelial surface reveals signs of a metaplastic change after 6 months of treatment with topically applied estriol. The cilia and goblet cells decreased and the nasal mucosa thickened. Also, some ciliated columnar epithelium transformed into a thick-layered, stratified keratinizing squamous epithelium (26). Based on these findings and knowledge of the ultrastructural changes of the nasal mucosa, we were interested to find out how these changes would impact the ciliary beating pattern of the nasal epithelial cell surface under topically applied estriol ointment. We were also eager to determine how the nasal mucosa of HHT patients differed from that of a normal, healthy control group, as data regarding the ciliary function of HHT patients is presently lacking.

Interestingly, after analyzing the harvested cilia, we detected an undisrupted ciliary carpet on the mucosal surface with an intact and uniform ciliary beat pattern in all three groups of patients. There were no statistically significant differences between these three groups. This is a very important finding, especially concerning the situation of the nasal mucosa of HHT patients who undergo not only several traumatic procedures of a nasal packing ${ }^{(35)}$ but also many intervals of a coagulation to control their recurrent episodes of epistaxis. All of these traumatic incidences can induce synechia or atrophy of the mucosa ${ }^{(36)}$, which can have a major impact on the ciliary function ${ }^{(37)}$. 


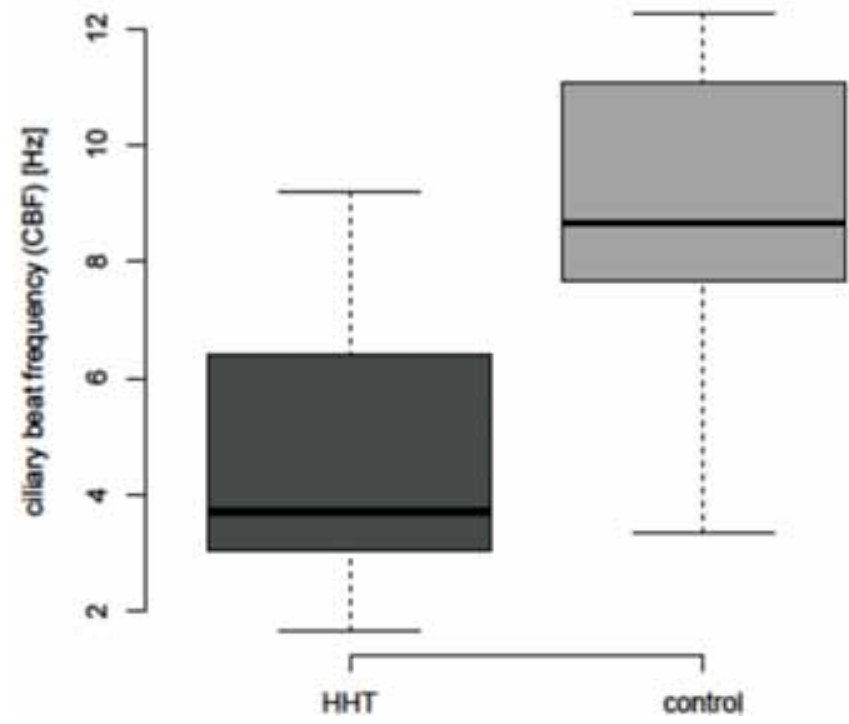

Figure 2. Box-Whisker Plot of the HHT and control group's CBF. The boxes represent the interquartile range (IQR) with the whiskers extending up to 1.5 times the IQR, the median is marked as a solid line.

\section{Ciliary beat frequency $(C B F)$}

The cilia in the healthy control group had a mean CBF of $9.12 \mathrm{~Hz}( \pm 2.43 \mathrm{~Hz})$, which is in line with previously published data obtained when working at room temperature conditions ${ }^{(38)}$. In HHT patients, the mean CBF of $4.75 \mathrm{~Hz}( \pm 2.23$ $\mathrm{Hz}$ ) was significantly reduced when compared to the healthy control group. An abnormal beating pattern seems to be a very unlikely reason for this phenomenon, as the VI index did not show any statistically significant differences amongst healthy controls and HHT patients using or not using estriol nose ointment. Even a physiological reaction of the mucosa to past mechanical stress from the recurrent episodes of epistaxis seems to be an unlikely cause, as mechanical stress activates mucus clearance by increasing the rates of ATP release into the luminal compartment. This ultimately results in an increase in airway surface liquid hydration. In this way, ciliated mucosa can respond to stress inducing stimuli on the intrapulmonary airways by stimulating secretion and accelerating clearance ${ }^{(39,40)}$. This leaves the idea of microscopically disrupted cells on the surface of the mucosa showing an intact beating pattern but a decreased, slower $\mathrm{CBF}^{(32)}$.

By comparing both the HHT groups with each other, it became obvious that the CBF with a mean beat frequency of $3.89 \mathrm{~Hz}$ in HHT patients using estriol nose ointment was significantly reduced compared to the $\mathrm{CBF}$ in HHT patients not using estriol nose ointment. Again, it was interesting to see that under topically applied estrogens, a ciliary beat still exists. It is not absent, as one might assume, in spite of the estriol induced epithelial metaplasia with a possible decrease in the amount of active cilia or possible elimination of ciliated cells, as suggested in previous ultrastructural studies ${ }^{(26)}$.

A major drawback of our study is the fact that we cannot make any statements on the ciliary function in vivo, such as the mucus transport time (MTT). Conducting these tests with HHT patients turned out to be extremely difficult due to their incrustation, synechia, and in some cases, septal perforations from multiple coagulation procedures. However, in a study published by Duchateau et al. in 1985, the authors concluded that the informative significance of CBF values $(\mathrm{Hz})$ is sufficient as there is a highly significant correlation between the CBF and the MTT ${ }^{(41)}$.

The preserved but impaired ciliary function in HHT patients may be important for proper respiratory defense mechanisms ${ }^{(27)}$. Further studies including questionnaire surveys are necessary to evaluate a possible effect of estriol ointments on nasal symptoms like aridity. An ideal setting of these studies would include measurements of the CBF and VI-Index before and after the application of estriol nose ointment to evaluate its effect on healthy mucosa and also the mucosa of HHT patients.

Cignarella et al., as well as Vase et al. proved that under the systemic effect of estrogens, the vessel wall integrity in patients with HHT shows substantial improvement without any signs of a reduction in the degree of the vessel dilatations. This emphasizes the fact that the nasal mucosa should be more robust against local trauma after using estriol ointment ${ }^{(42,43)}$. The fact that the newly formed squamous epithelium within the nose builds a cover over the nasal telangiectasia contributes to this trauma protection. 


\section{CONCLUSION}

$\mathrm{CBF}$ in HHT patients is significantly impaired compared to the CBF of the healthy control group. This impairment is even more distinct if HHT patients topically apply estriol as a nose ointment for more than 3 to 6 months. A ciliary beating pattern is found in HHT patients using estriol nose ointment in spite of the fact that estrogens are known to induce a transformation of the ciliated columnar epithelium into a keratinizing squamous epithelium. This data may justify the adjuvant application of estriol as a nose ointment in the treatment of epistaxis in HHT patients without the fear of damage to the mucus clearance within the nose.

\section{AUTHORSHIP CONTRIBUTION}

JUS: Experiments and writing of paper; BAS: Scientific and functional support; $\mathrm{CH}$ : Analysis of samples; SSK: Analysis of samples; KH: Founder and head of department; HS: Scientific and functional support.

\section{CONFLICT OF INTEREST}

There is no conflict of interest.

\section{REFERENCES}

1. Chapman KR, Allen LJ, Romet TT. Pulmonary function in normal subjects following exercise at cold ambient temperatures. Eur J Appl Physiol Occup Physiol. 1990; 60: 228-232.

2. Satir P, Sleigh MA. The physiology of cilia and mucociliary interactions. Annu Rev Physiol. 1990; 52: 137-155.

3. Hilfinger A, Chattopadhyay AK, Julicher F. Nonlinear dynamics of cilia and flagella. Phys Rev E Stat Nonlin Soft Matter Phys. 2009; 79(5 Pt 1): 051918

4. Stannard W, O'Callaghan C. Ciliary function and the role of cilia in clearance. J Aerosol Med. 2006; 19: 110-115.

5. Jorissen M, Bessems A. Influence of culture duration and ciliogenesis on the relationship between ciliary beat frequency and temperature in nasal epithelial cells. Eur Arch Otorhinolaryngol. 1995; 252: 451-454.

6. Shah AS, Ben-Shahar Y, Moninger TO, Kline JN, Welsh MJ Motile cilia of human airway epithelia are chemosensory. Science. 2009; 28; 325(5944): 1131-1134.

7. Chen B, Shaari J, Claire SE, et al. Altered sinonasal ciliary dynamics in chronic rhinosinusitis. Am J Rhinol. 2006; 20: 325-329.

8. Elliott MK, Sisson JH, Wyatt TA. Effects of cigarette smoke and alcohol on ciliated tracheal epithelium and inflammatory cell recruitment. Am J Respir Cell Mol Biol. 2007; 36: 452-459.

9. Sommer JU, Schafer K, Omran H, et al. ENT manifestations in patients with primary ciliary dyskinesia: prevalence and significance of otorhinolaryngologic co-morbidities. Eur Arch Otorhinolaryngol. 2011; 268(3): 383-388.

10. Weber FP. Multiple Hereditary Developmental Angeiomata (Telangiectases), with Recurring Haemorrhages. Proc R Soc Med. 1908; 1(Laryngol Sect): 43-44.

11. Osler W. On a family form of recurring epistaxis, associated with multiple telangiectases of the skin and mucous membranes. Bulletin of the Johns Hopkins Hospital. 1901; 12: 7.

12. Rendu H. Epistaxis repetees chez un sujet porteur de petits angiomes cutanes et muquez. Gazette des Hopitaux Civils et Militaires (Paris). 1896; 135: 3.

13. Braverman IM, Keh A, Jacobson BS. Ultrastructure and threedimensional organization of the telangiectases of hereditary hemorrhagic telangiectasia. J Invest Dermatol. 1990; 95: 422-427.

14. Faughnan ME, Palda VA, Garcia-Tsao G, et al. International guidelines for the diagnosis and management of hereditary haemorrhagic telangiectasia. J Med Genet. 2011; 14.
15. Shovlin CL, Guttmacher AE, Buscarini E, et al. Diagnostic criteria for hereditary hemorrhagic telangiectasia (Rendu-Osler-Weber syndrome). Am J Med Genet. 2000; 91: 66-67.

16. Bergler W, Riedel F, Baker-Schreyer A, Juncker C, Hormann K. Argon plasma coagulation for the treatment of hereditary hemorrhagic telangiectasia. Laryngoscope. 1999; 109: 15-20.

17. Zohar Y, Sadov R, Shvili Y, Talmi Y, Laurian N. Surgical management of epistaxis in hereditary hemorrhagic telangiectasia. Arch Otolaryngol Head Neck Surg. 1987; 113: 754-757.

18. Werner JA, Lippert BM, Geisthoff UW, Rudert H. Nd:YAG laser therapy of recurrent epistaxis in hereditary hemorrhagic telangiectasia. Laryngorhinootologie. 1997; 76: 495-501.

19. Fiorella ML, Ross D, Henderson KJ, White RI, Jr. Outcome of septal dermoplasty in patients with hereditary hemorrhagic telangiectasia. Laryngoscope. 2005; 115: 301-305.

20. Saunders WH. Septal dermoplasty for hereditary telangiectasia and other conditions. Otolaryngol Clin North Am. 1973; 6: 745755.

21. Fischer M, Dietrich U, Labisch C, Zanella FE, Jahnke K. Critical evaluation of vascular embolization in patients with Rendu-Osler disease. Laryngorhinootologie. 1997; 76: 490-494.

22. Lund VJ, Howard DJ. Closure of the nasal cavities in the treatment of refractory hereditary haemorrhagic telangiectasia. J Laryngol Otol. 1997; 111: 30-33.

23. Klepfish A, Berrebi A, Schattner A. Intranasal tranexamic acid treatment for severe epistaxis in hereditary hemorrhagic telangiectasia. Arch Intern Med. 2001; 12; 161: 767.

24. Bergler W, Sadick H, Gotte K, Riedel F, Hormann K. Topical estrogens combined with argon plasma coagulation in the management of epistaxis in hereditary hemorrhagic telangiectasia. Ann Otol Rhinol Laryngol. 2002; 111(3 Pt 1): 222-228.

25. Sadick H, Riedel F, Oulmi J, Hormann K, Bergler WF. Argon plasma coagulation and topically applied estriol. Long-term results in the treatment of hereditary hemorrhagic telangiectasia of the nasal mucosa. HNO. 2003; 51: 118-124.

26. Sadick H, Bergler WF, Oulmi-Kagermann J, et al. Estriol induced squamous metaplasia on the nasal mucosa in patients with hereditary hemorrhagic telangiectasia. Arch Med Res. 2005; 36: 468-473.

27. Proctor DF, Adams GK, Andersen I, Man SF. Nasal mucociliary clearance in man. Ciba Found Symp. 1978: 219-234.

28. Andersen I, Proctor DF. Measurement of nasal mucociliary clearance. Eur J Respir Dis Suppl. 1983; 127: 37-40.

29. Sommer JU, Gross S, Hormann K, Stuck BA. Timedependent changes in nasal ciliary beat frequency. Eur Arch Otorhinolaryngol. 2010; 267: 1383-1387.

30. Rollin M, Seymour K, Hariri M, Harcourt J. Rhinosinusitis, symptomatology and absence of polyposis in children with primary ciliary dyskinesia. Rhinology. 2009; 47: 75-78.

31. Sommer JU, Gross S, Hormann K, Stuck BA. Timedependent changes in nasal ciliary beat frequency. Eur Arch Otorhinolaryngol. 2010; 267: 1383-1387.

32. Thomas B, Rutman A, O'Callaghan C. Disrupted ciliated epithelium shows slower ciliary beat frequency and increased dyskinesia. Eur Respir J. 2009; 34: 401-404.

33. Sisson JH, Stoner JA, Ammons BA, Wyatt TA. All-digital image capture and whole-field analysis of ciliary beat frequency. J Microsc. 2003; 211: 103-111.

34. R-Development-Core-Team. R: A Language and Environment for Statistical Computing. Vienna, Austria: R Foundation for Statistical Computing; 2010.

35. Beule AG, Weber RK, Kaftan H, Hosemann W. Review: pathophysiology and methodology of nasal packing. Laryngorhinootologie. 2004; 83: 534-551; quiz 53-56.

36. Ferri E, Ianniello F, Armato E, Cavaleri S, Capuzzo P. The application of argon plasma coagulation (APC) in surgical treatment of inferior turbinates. Acta Otorhinolaryngol Ital. 2002; 22: 220-226.

37. Schrodter S, Biermann E, Halata Z. Histological evaluation of age-related changes in human respiratory mucosa of the middle turbinate. Anat Embryol. (Berl). 2003; 207: 19-27. 
38. Dimova S, Maes F, Brewster ME, Jorissen M, Noppe M, Augustijns P. High-speed digital imaging method for ciliary beat frequency measurement. J Pharm Pharmacol. 2005; 57: 521-526.

39. Button B, Boucher RC. Role of mechanical stress in regulating airway surface hydration and mucus clearance rates. Respir Physiol Neurobiol. 2008; 163: 189-201.

40. Tarran R, Grubb BR, Gatzy JT, Davis CW, Boucher RC. The relative roles of passive surface forces and active ion transport in the modulation of airway surface liquid volume and composition. J Gen Physiol. 2001; 118: 223-236.

41. Duchateau GS, Graamans K, Zuidema J, Merkus FW. Correlation between nasal ciliary beat frequency and mucus transport rate in volunteers. Laryngoscope. 1985; 95: 854-859.

42. Cignarella A, Paoletti R, Puglisi L. Direct effects of estrogen on the vessel wall. Med Res Rev. 2001; 21: 171-184.

43. Vase P. Estrogen treatment of hereditary hemorrhagic telangiectasia. A double-blind controlled clinical trial. Acta Med Scand.
1981; 209: 393-396.

\section{J. Ulrich Sommer, M.D.}

Department of Otorhinolaryngology Head and Neck Surgery

University Hospital Mannheim

Theodor-Kutzer-Ufer 1-3

68167 Mannheim

Germany

Tel: +49-(0)621-383 1600

Fax: +49-(0)621-383 3827

E-mail: ulrich.sommer@umm.de 